an excellent analysis using an effective field theory with a linear potential. A phenomenological model which attempts to describe charmed particle decays is presented by V. Mathur.

Each of the remaining three papers deals with areas with little connection to other work presented at the conference. In a discussion of covariance problems in two dimensions, C. Hagen questions the validity of light cone field theory. Deviations from scaling are analysed by $\mathbf{H}$. Politzer, using the renormalisation group and the Wilson operator product expansion. An unconventional model involving massive gluons and unconfined colour is presented by $R$. N. Mohapatra as a possible explanation of high $y$ anomalies and dimuon events. (It should be noted

\section{Fungal symbiosis}

The Biology of Symbiotic Fungi. By Roderic Cooke. Pp. xi+282. (Wiley: London and New York, 1977.) \$21; $£ 10.75$.

THE word Symbiotic in the title of this book is used in a much broader sense than most botanists have used it in recent years. Instead, the author adopts the older usage of de Bary, recently introduced again by $D$. $H$. Lewis, using the term symbiosis to include any association between two organisms which involves long-term intimate contact. This includes, therefore, both antagonistic (parasitic) relationships as well as the mutalistic relationships most commonly considered by botanists to be symbiosis. The author recognises that some relationships exist which cannot with certainty be classified as antagonistic or mutualistic, and these he calls neutral. Neutral symbioses are particularly important in associations of fungi with animals.

Symbiotic relationships between fungi and animals, fungi and higher plants, fungi and algae (lichens) and between fungi and other fungi, are all considered. As explained above, these are classified as antagonistic, neutral or mutualistic relationships; further, the fungi are divided on nutritional grounds, again following D. H. Lewis, into necrotrophic and biotrophic species. In this connection it may be mentioned that the author recognises - and this will be welcomed by many plant pathologists- that quite a number of plant-pathogenic fungi are not easily characterised as necrotrophs or biotrophs; some seem to start their association with their host plant as that recent experimental work, not yet made public at the time of publication of this collection, offers no evidence for the phenomena this paper attempts to explain.)

This book contains some excellent reports on theoretical work on the quark binding problem and field theory, and would be appropriate for the shelves of physics libraries. For those who are actively working in these areas, however, purchase of this book seems an expensive way to acquire papers which are readily available either as reprints or as published articles.

Larry McLerran

Larry McLerran is a research associate in the Laboratory for Nuclear Science, Massachusetts Institute of Technology, Cambridge, Massachusetts.

biotrophs, finishing up as necrotrophs, and these are clasified in this book as a distinct group, hemibiotrophs.

A very wide range of possible associations are thus considered; examples are discussed in each category with particular reference to nutritional and other physiological relationships between the two symbiotic associates. Five chapters are devoted to symbioses with animals and six chapters to symbioses between fungi and plants, including the lichen symbiosis. Since much more is known about fungusplant symbioses than fungus-animal symbioses, one might expect that the inevitably more 'concentrated' material which appears in the chapters dealing with plants would lead to a degree of imbalance. This is not the case; the book gives a strong impression of unity, and this is all the more valuable in that it covers a range of topics which have never before been brought systematically together.

Indeed, it is striking how similar, in many respects, the fungi involved in biotrophic associations with animals are to those similarly involved with plants. To take one example, it seems to be very common among biotrophs in both situations that they are difficult to culture axenically. This characteristic may become less noticeable as cultural techniques improve, but at present it is quite striking.

This is undoubtedly a valuable book covering a wide field. It will be of interest not only to mycologists, but also to all botanists and zoologists interested in fungal symbiosis. Not many students will be able to afford it let's hope that it is widely bought by departmental libraries. P. W. Brian

P. W. Brian has just retired as Professor of Botany at the University of Cambridge, $U K$

\section{Introducing cryobiochemistry}

Cryobiochemistry: An Introduction. By Pierre Douzou. Pp.x +286 . (Academic: London and New York, 1977.) £12.60; $\$ 24.65$.

Professor Douzou's book on the theory and practice of low temperature biochemistry appears at a time of increasing interest in the technique, and when the first reports of it being used at its full power are beginning to appear. The aim of the technique is no less than the thermal resolution of the individual steps in biochemical processes, especially enzyme reactions, and the stabilisation of intermediates for timescales sufficient for their detailed examination by the more powerful analytical techniques. This is an exciting prospect for the molecular biochemists, and this book is doubly welcome, because not only has Professor Douzou, as the major pioneer of the field, given us the gift of his ten years of experience and development of the subject, but he has also presented it in a well written and clearly organised text.

The major experimental problem of ensuring that even in solutions of mixed solvents at temperatures down to $-100^{\circ} \mathrm{C}$ the proteins are both structurally intact and functionally, potentially competent, is dealt with at length. The book also provides invaluable guidance in choosing solvent systems, the $p \mathrm{H}$, dielectric constant and viscosity at subzero temperatures of which are closely matched with those of the normal room temperature environment. In this respect, the book fulfills the function of a laboratory handbook on low temperature procedures, with many specific examples, drawings of special apparatus and tables of physical properties of mixed solvent systems. At the same time the book reveals its origins as a lecture course, in beginning each section at the most elementary level and taking the reader step by step, from the properties of mixed solvents, through their effects on enzyme activity, to the study of biological molecules at subzero temperatures.

This is a first-class introduction in both the theoretical and practical sense to what is bound to become an increasingly important technique in biochemistry.

C. C. F. Blake

C. C. F. Blake is Lecturer in Molecular Biophysics at the University of Oxford, $U K$. 